\title{
Etiología y factores pronósticos de la neumonía adquirida en la comunidad en el adulto hospitalizado, Puerto Montt, Chile
}

\author{
Raúl Riquelme $\mathbf{O}^{\mathbf{1}}$, Mauricio Riquelme $\mathbf{O}^{\mathbf{1}}$, \\ María Luisa Rioseco $Z^{2}$, Valeria Gómez $M^{1 a}$, \\ Rodrigo Gil $\mathrm{D}^{3}$, Antonio Torres $\mathrm{M}^{\mathbf{4}}$. \\ Etiology and prognostics factors of \\ community-acquired pneumonia \\ among adults patients admitted to \\ a regional hospital in Chile
}

Background: Community-acquired pneumonia (CAP) is a common and serious illness in Chile. Aim: To evaluate the etiology, severity, prognostic factors and blood culture yield of CAP requiring hospitalization in Puerto Montt in Southern, Chile. Patients and methods: All non immunocompromised adults with CAP admitted at Puerto Montt Hospital during one year, were prospectively studied. Clinical and radiological assessment was done in all patients. Blood and sputum cultures were obtained and serology for atypical agents was determined. Results: We studied 200 patients, aged $63 \pm 19$ years (109 males). The prognostic factors associated with mortality were an age over 65 years, an altered mental status, shock and acute renal failure. Etiology was demonstrated in $29 \%$ of patients. The most frequent pathogens were Streptococcus pneumoniae (40.7\%), Haemophilus influenzae (23.7\%) and Chlamydia pneumoniae (16.9\%). Mixed infections were found in $17 \%$. Fifteen atypical pathogens were identified in 12 patients. Of these only two received a specific treatment but no one died and their length hospital stay was similar than in the rest of the patients. Overall, blood cultures were positive in $12.5 \%$ of patients, but among alcoholics, $58 \%$ were positive. In only one percent of cases, positive cultures motivated therapeutic changes among clinicians. Eight percent of S pneumoniae strains were penicillin resistant. Conclusions: Atypical agents were a common cause of CAP in this group of patients, but their pathogenic role and treatment requirements are debatable. Focusing blood cultures on specific groups could improve their yield. The rate of Penicillin resistance for Spneumoniae was low (Rev Méd Chile 2006; 134: 597-605).

(Key words: Haemophilus influenzae; Penicillin resistance; Pneumonia, bacterial; Streptococcus pneumoniae)

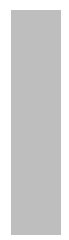

Recibido el 9 de mayo, 2005. Aceptado el 3 de noviembre, 2005.

Trabajo financiado parcialmente por la Sociedad Chilena de Enfermedades Respiratorias y RedRespira-ISCIII-RTIC-03/11.

${ }^{1}$ Servicio de Medicina Interna y ${ }^{2}$ Laboratorio de Microbiología del Hospital de Puerto Montt, Chile. ${ }^{3}$ Instituto Nacional del Tórax, Chile. ${ }^{4}$ Servicio de Neumología, Hospital Cliníc, Barcelona, España.

aEnfermera universitaria

Correspondencia a: Dr. Raúl Riquelme Oyarzún. Bellavista 123, of. 402, Puerto Montt, Chile. Teléfono-fax: 5665 289409. E mail: raul@telsur.cl 
Los patrones microbiológicos señalados en diferentes estudios de etiología de la neumonía adquirida en la comunidad (NAC), en el paciente inmunocompetente que se hospitaliza, difieren significativamente ${ }^{1}$. Esto se debe a numerosos factores, incluyendo diferencias en las características de la población examinada, extensión del estudio microbiológico realizado, localización geográfica y los criterios diagnósticos. En Chile, los estudios microbiológicos han sido escasos, especialmente con respecto a los patógenos atípicos (Mycoplasma pneumoniae, Chlamydia pneumoniae y Legionella pneumophila) ${ }^{2-7}$. Varias publicaciones internacionales han señalado que el tratamiento de estas infecciones debe cubrir siempre estos microorganismos ${ }^{8-10}$. El Consenso Chileno de Neumonía Comunitaria, basado en criterios de edad, comorbilidad y gravedad de la enfermedad, no recomienda como primera elección un tratamiento específico para agentes atípicos, excepto en el paciente con NAC grave, que requiere tratamiento en Unidades de Cuidado Intensivo $(\mathrm{UCI})^{11}$. El conocimiento de los agentes etiológicos predominantes de las NAC es fundamental para orientar decisiones terapéuticas empíricas, y es evidente que recomendaciones basadas en estudios nacionales tienen un mayor valor que aquellas basadas en publicaciones extranjeras.

Presentamos un estudio prospectivo de pacientes adultos no inmunocomprometidos que fueron hospitalizados por NAC en el período de un año en el Hospital de Puerto Montt, tratando de determinar el espectro microbiano local, su asociación con la gravedad y la presencia de comorbilidades específicas, y los factores pronósticos asociados a evolución clínica desfavorable en el hospital.

\section{PACIENTES Y MÉTODOS}

En el Hospital de Puerto Montt, X región, hospital general de 400 camas, entre el 1 de junio de 2000 y 31 mayo de 2001, estudiamos prospectivamente todos los pacientes mayores de 15 años de edad admitidos en cualquier servicio del hospital por un episodio de NAC, que cumpliera con los criterios diagnósticos de Fang y cols ${ }^{12}$. Se excluyeron aquellos con antecedentes de hospitalización previa (últimos 30 días), VIH-1 positivos, neopla- sia activa, neutropenia (recuento glóbulos blancos (GB) $<1,000$ por $\mathrm{mm}^{3}$ ), tratamiento quimioterápi$\mathrm{co}$, inmunosupresor 0 con esteroides $(\geq 20 \mathrm{mg}$ prednisona al día).

Colección de datos. Las variables registradas fueron edad, sexo, comorbilidades (cardiopatía, diabetes mellitus, EPOC, asma bronquial, insuficiencia renal crónica, cirrosis u otra enfermedad hepática crónica, cáncer, bronquiectasias o secuelas TBC), alcoholismo, tabaquismo, terapia antimicrobiana previa (últimos 15 días), lugar de residencia, sospecha de aspiración, síntomas clínicos, signos vitales y resultados de exámenes de laboratorio (recuento de GB, gases arteriales, uremia, creatininemia, electrolitograma, albúmina plasmática). Se tomó radiografía de tórax a todos los pacientes. Se consignó el esquema antibiótico usado, las modificaciones y las razones del cambio. También se registró si hubo insuficiencia renal aguda, necesidad de UCI, ventilación mecánica (VM), mortalidad y si ésta pudo ser atribuida a la NAC. Se definieron shock e insuficiencia renal aguda según criterios previamente publicados ${ }^{13}$. Alcoholismo se definió como ingestión estimada $\geq 80$ gramos de alcohol por día en el último año. El modelo predictivo de Fine y $\mathrm{col}^{14} \mathrm{se}$ aplicó en todos los pacientes.

La evaluación microbiológica al ingreso incluyó tinción de Gram y cultivo de esputo, dos hemocultivos (HCs) y estudio de líquido pleural si había derrame. Además, se tomaron muestras de sangre para serologías al ingreso, que se repitieron 15 a 20 días después; las muestras pareadas se procesaron usando immunofluorescencia indirecta para $M$ pneumoniae (Zeus ${ }^{\circledR}$ ), C pneumoniae (Focus ${ }^{\circledR}$ ) y L pneumophila serogrupos 1-4 (Zeus $\left.{ }^{\circledR}\right)$. En todas las bacterias potencialmente patógenas aisladas, se realizaron pruebas de susceptibilidad antimicrobiana con la técnica de difusión en agar según las recomendaciones del National Committee for Clinical Laboratory Standards ${ }^{15}$. Los antibióticos evaluados en las cepas de $\mathrm{S}$ pneumoniae fueron oxacilina, eritromicina, cloramfenicol, rifampicina, vancomicina y levofloxacina. Para las cepas resistentes a oxacilina, se determinó la concentración inhibitoria mínima (CIM), para penicilina y ceftriaxona mediante epsilometría (E-test). En las cepas de $\mathrm{H}$ influenzae, se evaluaron ampicilina, cloramfenicol, ciprofloxacina, ceftriaxona, cefuroxima y trimetroprim/sulfametoxazol. 
El diagnóstico etiológico se consideró definitivo si el microorganismo aislado provenía de una muestra estéril (sangre, líquido pleural) o si había seroconversión (aumento $\geq 4$ veces en el título de IgG sérica entre la fase aguda y la de convalescencia) y presuntivo cuando hubo desarrollo de patógenos en muestra válida de esputo ( $>25$ neutrófilos polimorfonucleares y $<10$ células epiteliales por campo) o si la serología mostraba un título sostenidamente alto, sin variación en las muestras pareadas. Esos títulos fueron para $\mathrm{M}$ pneumoniae IgG $\geq 1: 128$; C pneumoniae IgG $\geq 1: 520$ y L pneumophila serogrupos $1-4$ IgG $\geq 1: 128$.

Estadística. Los resultados son expresados como valores promedio $\pm \mathrm{DE}$. Para comparar las variables continuas se usó test $\mathrm{t}$ de Student y el test de Chi cuadrado o test exacto de Fisher cuando fue apropiado para las variables categóricas. Para evaluar los factores de riesgo asociados a mortalidad se aplicó un modelo de regresión logística. La variable dependiente fue la letalidad de la neumonía en el hospital. Aquellas variables con un valor de $\mathrm{p}<0,10$ o que eran biológicamente plausibles se aceptaron para el análisis multivariado. Se consideró significativo un valor de $\mathrm{p}<0,05$. Los datos fueron analizados usando el paquete estadístico SPSS para Windows ${ }^{\circledR}, 10.0$ (SPSS, Chicago, IL, USA).

\section{RESULTADOS}

Se evaluaron 200 pacientes con NAC, la edad media fue $63 \pm 19$ años, $74,5 \%$ tenía una o más comorbilidades. Las características clínicas generales de la población se describen en la Tabla 1. Los

Tabla 1. Características clínicas al ingreso de 200 pacientes hospitalizados por neumonía comunitaria, H ospital de Puerto M ontt, C hile

\begin{tabular}{|lrc|}
\hline Hallazgos clínicos & $\mathrm{N}$ & $\%$ \\
\hline Edad (años \pm DE) & $63 \pm 19$ & 57,5 \\
Sexo masculino & 109 & 2 \\
Procedencia de hogar de ancianos & 4 & 74,5 \\
Comorbilidad $\geq 1$ & 149 & 33,5 \\
EPOC/asma & 67 & 20,5 \\
Cardiopatía & 41 & 16 \\
Diabetes mellitus & 32 & 11 \\
Secuelas de TBC o bronquiectasias & 22 & 10 \\
Alcoholismo & 20 & 7 \\
Enfermedad cerebrovascular & 14 & 7 \\
Insuficiencia renal crónica & 14 & 5 \\
Enfermedad hepática crónica & 10 & 13,5 \\
Tabaquismo & 27 & 20,5 \\
Ex fumadores & 41 & 8,5 \\
Sospecha de aspiración & 17 & 27 \\
Compromiso de conciencia & 54 & 65 \\
Fiebre en primeras 48 h & 130 & 81 \\
Tos & 162 & 76,5 \\
Expectoración & 153 & 67,5 \\
Disnea & 135 & 38 \\
Dolor torácico & 76 & 25,5 \\
Calofríos & 51 & 12,5 \\
Recibieron antibióticos antes de ingresar (15 días) & 24 & 49,5 \\
Frecuencia respiratoria $>30$ x' & 99 & \\
\hline
\end{tabular}


principales hallazgos radiológicos, de laboratorio y la evolución intrahospitalaria se describen en la Tabla 2. La estadía promedio en el hospital fue $12 \pm 9,6$ días y la letalidad $17,5 \%$.

El tratamiento antibiótico prescrito en la admisión incluyó un agente betalactámico solo (penicilina sódica, ceftriaxona, ampicilina o cloxacilina) 0 combinado en $94 \%$ de los casos. Sólo en 30 casos (15\%) se empleó un antibiótico con cobertura para patógenos atípicos. En 83 pacientes hubo cambio en el esquema antibiótico (Tabla 2). Hubo un caso en que la terapia empírica inicial no cubrió el agente etiológico aislado y el paciente falleció (ampicilina/S aureus).
Fallecieron 35 pacientes, de ellos, 42,8\% durante los primeros 5 días de hospitalización (15/35 vs $20 / 35 p=0,001$ ). En aquellos que fallecieron, el tratamiento empírico cubrió los microorganismos atípicos sólo en 4 casos $(11,4 \%)$.

Los principales factores de mal pronóstico en la admisión al hospital fueron edad $\geq 65$ años $(p=0,005)$, confusión mental $(p=0,005)$, ausencia de fiebre en las primeras $48 \mathrm{~h}(\mathrm{p}=0,002)$ y durante la evolución hospitalaria el desarrollo de insuficiencia renal aguda $(p=0,001)$ y shock séptico $(\mathrm{p}=0,001)$ (Tabla 3).

La distribución de las técnicas diagnósticas no tuvo sesgos por grupos de edad o comorbilidades

Tabla 2. Exámenes de laboratorio, radiología y evolución intrahospitalaria de $\mathbf{2 0 0}$ casos de neumonía comunitaria, H ospital de Puerto M ontt, Chile

\begin{tabular}{|lrc|}
\hline & $\mathrm{N}$ & $\%$ \\
\hline Leucocitos $>20.000{\text { por } \mathrm{mm}^{3}}^{3}$ & $30 / 200$ & 15 \\
Leucocitos <4.000 por mm ${ }^{3}$ & $5 / 200$ & 2,5 \\
PaO2/FiO2 <250 & $58 / 186$ & 31,2 \\
Infiltrados radiológicos alveolares & $129 / 200$ & 64 \\
Infiltrados radiológicos bilaterales & $51 / 200$ & 34 \\
Compromiso mayor de 1 lóbulo & $68 / 200$ & 34 \\
Aumento de infiltrados radiológicos en 50\% (primeras 48 h) & $9 / 200$ & 4,5 \\
Derrame pleural & $14 / 200$ & 7 \\
Complicación pleural & $6 / 200$ & 3 \\
Requirió UCI* & $18 / 200$ & 9 \\
Requirió ventilación mecánica & $15 / 200$ & 7,5 \\
Shock & $9 / 200$ & 4,5 \\
Insuficiencia renal aguda & $12 / 200$ & 6 \\
Infección extrapulmonar & $7 / 200$ & 3,5 \\
Cambio de antibióticos durante hospitalización & $83 / 200$ & 41,5 \\
Cambio de antibióticos por deterioro clínico & $19 / 83$ & 22,9 \\
Cambio de antibiótico por determinación de etiología & $6 / 83$ & 7,2 \\
Cambio de antibiótico debido a resultado de hemocultivos & $1 / 21$ & 4,8 \\
Cumplieron recomendaciones terapéuticas de SER** & $59 / 200$ & 29,5 \\
Letalidad hospitalaria categoría de Fine I y II & $0 / 33$ & 0 \\
Letalidad hospitalaria categoría de Fine III & $2 / 34$ & 5,9 \\
Letalidad hospitalaria categoría de Fine IV & $11 / 76$ & 14,5 \\
Letalidad hospitalaria categoría de Fine V & $22 / 57$ & 38,6 \\
Letalidad hospitalaria & $35 / 200$ & 17,5 \\
Letalidad atribuible a NAC & $26 / 200$ & 13 \\
\hline
\end{tabular}

UCI: Unidad Cuidados Intensivos. **SER: Sociedad Chilena de Enfermedades Respiratorias. 


\section{Tabla 3. Factores de riesgo de muerte en adultos inmunocompetentes hospitalizados por neumonía comunitaria. Análisis uni y multivariado}

\begin{tabular}{|lrrccc|}
\hline & \multicolumn{2}{c}{ Univariado } & & \multicolumn{2}{c|}{ Multivariado } \\
& OR & IC $95 \%$ & $\mathrm{p}$ & OR & IC $95 \%$ \\
\hline Edad $\geq 65$ años & 3,4 & $1,5-7,9$ & 0,005 & 10,4 & $1,34-80,9$ \\
Confusión mental & 7,0 & $3,2-15,4$ & 0,001 & NS & \\
Requiere UCI & 4,6 & $1,7-12,7$ & 0,005 & NS & \\
Ventilación mecánica & 12,8 & $4,0-40,6$ & 0,001 & NS & \\
Shock & 20,4 & $4,0-103,1$ & 0,001 & NS & \\
Insuficiencia renal aguda & 32,6 & $6,7-57,6$ & 0,001 & 20,8 & $2,8-151,3$ \\
Frecuencia respiratoria $\geq 30$ resp/min & 2,6 & $1,2-5,7$ & 0,015 & NS & \\
Presión sistólica $\leq 100 \mathrm{mmHg}$ & 3,4 & $1,5-7,5$ & 0,004 & NS & \\
Nitrógeno ureico $\geq 40 \mathrm{mg} / \mathrm{dL}$ & 2,7 & $1,1-6,3$ & 0,022 & NS & \\
pH arterial $\leq 7,2$ & 4,2 & $1,2-14,6$ & 0,031 & NS & \\
PaO 2 FIO $2 \leq 250$ & 2,7 & $1,3-5,8$ & 0,013 & NS & \\
Albúmina sérica $\leq 3,4 \mathrm{~g} / \mathrm{dL}$ & 2,7 & $1,3-5,7$ & 0,015 & NS & \\
Ausencia de fiebre en primeras $48 \mathrm{~h}$ & 3,3 & $1,6-7,1$ & 0,002 & 2,9 & $1,0-7,6$ \\
Cambio antibiótico por deterioro clínico & 3,2 & $1,2-8,8$ & 0,050 & NS & \\
Indice de Fine $\geq 90$ & 10,7 & $2,5-46,2$ & 0,001 & NI & \\
\hline
\end{tabular}

OR=Odds ratio. $\mathrm{IC}=$ intervalo de confianza. $\mathrm{NS}=$ no significativo. $\mathrm{NI}=$ no incluido en análisis.

y se aislaron 69 microorganismos en 59 pacientes (29,5\%) (Tabla 4). En 12 pacientes, se identificaron 15 patógenos atípicos $(21,7 \%)$ y sólo 2 de ellos recibieron terapia específica (eritromicina), pero ninguno de ellos falleció o tuvo una hospitalización más prolongada. En 21 pacientes se detectó bacteriemia: $14 \mathrm{~S}$ pneumoniae, 4 Enterobacteriaceae y $3 \mathrm{~S}$ aureus. En los pacientes alcohólicos hubo mayor frecuencia de bacteriemia que en los no alcohólicos (41,2\% vs 9,3\%, $\mathrm{p}=0,002)$ y esto también ocurrió en los pacientes menores de 65 años ( $\mathrm{p}=0,02)$, pacientes con taquicardia mayor de $120 \mathrm{lat} / \mathrm{min}$ al ingreso $(\mathrm{p}=0,005)$ y con recuento de GB $<4.000 / \mathrm{mm}^{3}(\mathrm{p}=0,001)$. No se encontró asociación entre bacteriemia y mortalidad, pero los pacientes con hemocultivo positivo tuvieron hospitalizaciones más prolongadas $(17,8 \pm 15$ vs $11,5 \pm 8,5$ días, $p=0,005)$. Los médicos tratantes realizaron modificaciones del tratamiento antimicrobiano basados en el resultado de los hemocultivos sólo en $4,8 \%$ de los casos (1/21).

Una cepa de $\mathrm{S}$ pneumoniae $(4,2 \%)$ fue resistente a la penicilina con $\mathrm{CIM}=2 \mu \mathrm{g} / \mathrm{mL}$ y hubo otra con sensibilidad intermedia y que además era resistente a eritromicina. Hubo una cepa de $\mathrm{H}$ influenzae resistente a ampicilina (7,1\%) y 5 resistentes a cotrimoxazol (35,7\%).

Los principales patógenos respiratorios aislados según comorbilidad se describen en la Tabla 5. La etiología fue demostrada en $63,6 \%$ de los pacientes con secuelas de tuberculosis o bronquiectasias, comparado con $25,8 \%$ de aquellos sin estas patologías (OR 5,17 IC 95\% 2,0-13,1, $\mathrm{p}=0,001)$, principalmente en esputo $(78,6 \%)$ y sólo 2 casos por HCs. En los pacientes alcohólicos, la etiología fue demostrada en $60 \%(12 / 20)$ vs $26,1 \%$ (47/180) (OR 4,2 IC 95\% 1,6-11,0, $\mathrm{p}=0,003$ ), principalmente por HCs $(58,3 \%)$ y cultivo de esputo (33,3\%); en este grupo fue más probable tener una infección neumocócica bacteriémica.

Los casos con etiología demostrada se distribuyeron uniformemente en las cinco categonías de Fine. Si comparamos los grupos más leves con los grupos de mayor severidad y mortalidad (índice de Fine $\leq 89$ vs $\geq 90$ ) no encontramos diferencias en la frecuencia de las formas bacteriémicas ni en la frecuencia de 
Tabla 4. Etiología de la neumonía comunitaria del adulto hospitalizado según exámenes microbiológicos, H ospital de Puerto M ontt ( $N=59 / 200=29,5 \%$ )

\begin{tabular}{|c|c|c|c|c|c|c|}
\hline Patógeno & $\mathrm{N}$ & $\%$ & $\begin{array}{c}\text { Hemocultivos } \\
\mathrm{N}\end{array}$ & $\begin{array}{l}\text { Esputo } \\
\mathrm{N}\end{array}$ & $\begin{array}{c}\text { Serología } \\
\mathrm{N}\end{array}$ & $\begin{array}{c}\text { Pleura } \\
\mathrm{N}\end{array}$ \\
\hline Streptococcus pneumoniae & 24 & 40,7 & 14 & 9 & & 1 \\
\hline Haemophilus influenzae & 14 & 23,7 & & 14 & & \\
\hline Chlamydia pneumoniae & 10 & 16,9 & & & 10 & \\
\hline Bacilos Gram negativos & 8 & 13,6 & 4 & 4 & & \\
\hline Staphylococcus aureus & 7 & 11,9 & 3 & 4 & & \\
\hline Legionella pneumophila & 3 & 5,1 & & & 3 & \\
\hline Mycoplasma pneumoniae & 2 & 3,4 & & & 2 & \\
\hline Streptococcus milleri & 1 & 1,7 & & & & 1 \\
\hline Total & 69 & & 21 & 31 & 15 & 2 \\
\hline Casos mixtos* & 10 & 16,9 & & & & \\
\hline Diagnóstico certeza & 32 & 46 & 21 & & 9 & 2 \\
\hline Diagnóstico presuntivo & 37 & 54 & & 31 & 6 & \\
\hline Muestras $(+) /$ total & & & $21 / 168$ & $31 / 85$ & $15 / 60$ & \\
\hline Rendimiento exámenes, \% & & & 12,5 & 36,5 & 25 & \\
\hline
\end{tabular}

*S pneumoniae + Haemophilus (3); Mycoplasma + Chlamydia (2); Legionella + Chlamydia (1); Haemophilus + Chlamydia (1); Gram negativo + Chlamydia (1); S pneumoniae + Chlamydia (1); Saureus + Gram Negativo (1). Tabla 5. Etiología de la neumonía del adulto hospitalizado según comorbilidad
\[ N=59 / 200(29,5 \%) \]

\begin{tabular}{|lccccc|}
\hline & $\begin{array}{c}\text { Asma/EPOC } \\
\%\end{array}$ & $\begin{array}{c}\text { Cardiopatía } \\
\%\end{array}$ & $\begin{array}{c}\text { Secuela TBC } \\
\%\end{array}$ & $\begin{array}{c}\text { Alcoholismo } \\
\%\end{array}$ & $\begin{array}{c}>65 \text { años } \\
\%\end{array}$ \\
\hline S pneumoniae & 21,4 & 21,4 & 28,6 & 50,0 & 28,6 \\
H influenzae & 28,6 & 35,7 & 42,8 & 8,3 & 39,2 \\
C pneumoniae & 21,4 & 7,1 & - & 8,3 & 6,7 \\
Gram negativos & 10,7 & 14,2 & 21,4 & 16,6 & 17,8 \\
S aureus & 7,1 & 14,2 & 7,1 & 16,6 & 10,7 \\
L pneumophila & 7,1 & 7,1 & 7,1 & - & 3,4 \\
M pneumoniae & 7,1 & - & 14,2 & 8,3 & 1,6 \\
Etiología mixta & 33,3 & 8,3 & 21,4 & 16,6 & 25 \\
\hline
\end{tabular}

algún microorganismo en particular. Sin embargo, los bacilos Gram negativos (BGN) fueron más frecuentes en los casos más graves (7 vs 1$)$ y los atípicos en las formas leves (42,1\% vs $14,9 \%)$, aunque estas diferencias no fueron significativas.
DisCUSIÓN

Los principales hallazgos de este estudio son describir los factores de riesgo para mortalidad y darnos una visión del espectro microbiológico 
local de la NAC en el paciente adulto immunocompetente que se hospitaliza.

Es de gran importancia que en la evaluación inicial de un paciente con NAC se identifiquen aquellos factores asociados a un mayor riesgo de morir. Se han descrito varios índices pronósticos, destacando el de Fine y cols ${ }^{14}$ para la pesquisa de pacientes de bajo riesgo y que ha sido validado en el medio nacional en adultos inmunocompetentes hospitalizados por NAC ${ }^{3,16,17}$. Nuestro estudio no difiere de estos estudios nacionales y es llamativa la coincidencia con los factores contemplados en el criterio de la British Thoracic Society, denominado CURB por sus siglas en inglés (Confusion, Urea, Respiratory rate, Blood pressure). Este criterio permite la pesquisa de pacientes de alto riesgo con NAC grave y se correlaciona bien con mortalidad, alcanzando $2,4 \%$ en caso de ausencia de estos elementos, $8 \%$ con uno de ellos, $23 \%$ con dos, 33\% con tres y $83 \%$ con cuatro factores de riesgo ${ }^{10}$. Creemos que instrumentos simples, que permitan evaluar la gravedad de los pacientes con NAC en los servicios de urgencia y consultorios de atención primaria, debieran ser utilizados rutinariamente como ya ha sido propuesto en nuestro medio $^{16}$.

La información en Chile es escasa, especialmente en lo referente a los «gérmenes atípicos»-7.

Basado en una revisión de 15 estudios, Bartlett y col concluyen que S pneumoniae es el microorganismo más comúnmente identificado (20-60\%), seguido por $\mathrm{H}$ influenzae, S aureus, bacilos gram negativos, Legionella, $\mathrm{M}$ pneumoniae y $\mathrm{C}$ pneumoniae ${ }^{18}$. Nuestros datos (Tabla 4) coinciden con los reportados en la literatura. Ha sido difícil definir la importancia de los microorganismos atípicos y la necesidad de terapia específica. En las guías clínicas internacionales, se recomienda que las infecciones por patógenos atípicos sean consideradas en las diferentes categorías de riesgo ${ }^{8-10}$. Esto se basa en la posibilidad de que estos agentes sean responsables de alrededor de 10$20 \%$ de los casos de $\mathrm{NAC}^{18,19}$ y que la evolución de los pacientes es mejor si se incluyen macrólidos en el esquema terapéutico ${ }^{20-22}$. Sólo 2 de nuestros 15 enfermos recibieron tratamiento específico, pero esto no prolongó la hospitalización y ningún paciente falleció. En las infecciones mixtas, bastó tratar el otro microorganismo para que el paciente mejorara, sugiriendo un rol de acom- pañante para el agente atípico y creemos posible que en los casos en que se aisló exclusivamente un patógeno atípico, el agente principal no fue pesquisado. La Legionella puede producir un amplio espectro de síntomas que van desde tos ligera hasta una neumonía grave y también se han descrito casos en los cuales el paciente se recupera a pesar de un tratamiento con drogas consideradas inefectivas para ella ${ }^{23}$. De este modo, podemos afirmar que existen infecciones por agentes atípicos que no requieren tratamiento específico, pero debemos señalar que su importancia en el paciente grave pudo haber sido subestimada por la mortalidad precoz, que impidió la recolección de una segunda muestra para serología. El 88,6\% de los pacientes fallecidos no recibieron cobertura antibiótica para microorganismos atípicos y esto nos sugiere que la mejor evolución observada en los pacientes con NAC tratados con macrólidos ${ }^{20-22}$, refleja principalmente la efectividad sobre el grupo de mayor riesgo y gravedad. Así, nos parece razonable suponer que si este grupo de NAC grave hubiese recibido tratamiento combinado (ß-lactámico + macrólido), habría probablemente mejorado su pronóstico. Una aproximación al problema sería cubrir siempre los agentes atípicos en el grupo de mayor riesgo, pero no necesariamente en los casos leves ni en aquellos en que la participación de los atípicos es menos probable (sospecha de aspiración) o incluso en los casos donde exista una comorbilidad fuertemente asociada a otros patógenos, como por ejemplo, el alcoholismo.

En el manejo de los antimicrobianos, el juicio clínico ha demostrado ser más importante que los resultados de los exámenes microbiológicos y, en general, existe resistencia entre los médicos para modificar los esquemas empíricos por otros de menor espectro 24 . Obtuvimos HCs en $84 \%$ (168/ 200) de los pacientes, con un rendimiento de $12,5 \%$. Sin embargo, sólo en 1/168 (0,6\%) hubo modificación del esquema antimicrobiano basado en el informe del HC. Este resultado es algo menor que lo reportado por Chalasani $(1,4 \%)$ y Waterer $(1,7 \%)$ y muy similar a lo comunicado en otro estudio nacional $(0,4 \%)^{25-27}$. Basados en su escasa contribución al manejo clínico nos parece innecesario su uso rutinario en todos los pacientes con NAC que requieren hospitalización, reservándolos para los pacientes con NAC grave, inmuno- 
deprimidos, insuficiencia renal crónica y alcohóli$\cos ^{24}$. En los pacientes alcohólicos identificamos el agente etiológico en $60 \%$ de los casos, principalmente en base a los HCs siendo S pneumoniae el agente dominante. La focalización de los HCs a grupos específicos con mayor probabilidad de bacteriemia, mejoraría la relación costo/beneficio y es así como Metersky 28 identificó factores predictores de bacteriemia en NAC, creando un modelo que permitió reducir significativamente el número de HCs y aun así, detectar casi $90 \%$ de las bacteriemias.

En los pacientes con secuelas de TBC 0 bronquiectasias, la etiología se demostró con mayor frecuencia, pero principalmente con el estudio de la expectoración, resultado esperado porque se trata de pacientes que generalmente tienen abundante esputo y patógenos fácilmente detectables.

Consideramos que en nuestra región, con bajos porcentajes de $\mathrm{S}$ pneumoniae resistente a

\section{REFERENCIAS}

1. Moreno R, Riguelme R. Etiología de la neumonía adquirida en la comunidad en el adulto inmunocompetente. Rev Chil Enf Respir 2005; 21: 81-8.

2. Trucco O, Vicencio M, Salamanca L, Ojeda A, Oyonarte M, Prado V. Participación de Legionella pneumophila en neumonía extrahospitalaria del adulto en Santiago. Rev Chil Infect 1993; 10: 8995.

3. Saldías $F$, Mardónez JM, Marchesse $M$, Viviani $P$, FARIAS G, Díaz A. Neumonía adquirida en la comunidad en el adulto hospitalizado. Cuadro clínico y factores pronósticos. Rev Méd Chile 2002; 130: 1373-82.

4. Díaz A, Fuentes G, Couble B, Uribe R, Mercado G, Soza A ET AL. Etiología de la neumonía adquirida en la comunidad en adultos hospitalizados en Santiago, Chile: implicancias para la guías clínicas. Rev Chil Enf Respir 2005; 21: 23-32.

5. Lobos $\mathrm{T}$, Moreno $\mathrm{R}$, Carstens $\mathrm{M}$, Domenech $\mathrm{A}$, Piemonte P, Julet C et al. Seroprevalencia de infección por Legionella pneumophila en adultos sanos de Santiago de Chile. Rev Méd Chile 1993; 121: 1123-7. penicilina, aún no es necesario incluir la resistencia dentro de los criterios principales a considerar para definir esquemas terapéuticos. Esta realidad, resulta un estímulo para el uso racional de los antimicrobianos, como ocurre en Holanda y Alemania $^{9}$ evitando el aumento descontrolado de la resistencia a los antimicrobianos.

Concluimos que las NAC tienen distintos patrones etiológicos, algunos predeterminados por las comorbilidades y que los microorganismos atípicos son frecuentes pero la necesidad de tratarlos siempre es aún motivo de controversia. La relación costo beneficio de los HCs mejoraría si se solicitan en grupos específicos. El uso racional de los antibióticos se justifica no sólo por razones económicas sino por el desafío que implica buscar la mayor eficacia minimizando el riesgo de la selección de patógenos resistentes. En la elaboración de recomendaciones de tratamiento antimicrobiano en la NAC del adulto se deben considerar aspectos regionales y locales.

6. Lobos T, Saldías F, Cartagena C, Jover E, Alvarez M, Moreno R. Chlamydia pneumoniae en pacientes con neumonías adquiridas en la comunidad en Santiago de Chile. Rev Méd Chile 1998; 126: 14839.

7. Lobos T, Moreno R, Saldías F, Cartagena C, Ferres M. Seroprevalencia de infección por Chlamydia pneumoniae en población sana de Santiago de Chile. Rev Chil Enf Respir 1996; 12: 145-50.

8. Niederman MS, Mandell LA, Anzueto A, Bass JB, Broughton WA, CAmpbell GD et al. Guidelines for the management of adults with community-acquired pneumonia. Diagnosis, assessment of severity, antimicrobial therapy and prevention. Am J Respir Crit Care Med 2001; 163: 1730-54.

9. Bartlett J, Dowell S, Mandell L, File T, Musher D, FINE M. Practice guidelines for the management of community-acquired pneumonia in adults. Clin Infect Dis 2000; 31: 347-82.

10. British Thoracic Society Standards of Care Committee. British Thoracic Society guidelines for the management of Community-acquired Pneumonia in adults. Thorax 2001; 56 (suppl IV): 1-64.

11. Díaz A, Labarca J, Pérez C, Ruiz M, Wolff M. Tratamiento de la neumonía del adulto adquirida 
en la comunidad. Rev Chil Enf Respir 2005; 21: 117-31.

12. Fang GD, Fine M, Orloff J, Arisumi D, Yu VL, KAPOOR W ET AL. New emerging etiologies for community-acquired pneumonia with implications for therapy. Medicine 1990; 69: 307-16.

13. American Colegge of Chest Physicians, Society of Critical Care Medicine consensus conference commTEE. Definitions for sepsis and organ failure and guidelines for the use of innovative therapies in sepsis. Critical Care Med 1992; 20: 864-73.

14. Fine M, Auble T, Yealy D, Hanusa B, Weissfeld L, SINGER D ET AL. A prediction rule to identify lowrisk patients with community-acquired pneumonia. N Engl J Med 1997; 336: 243-50.

15. National Committee for Cunical Laboratory StanDARDS. Performance standards for antimicrobial susceptibility testing; twelfth informational supplement. 2002; $22 \mathrm{~N}^{\circ} 1$.

16. Saldías F, Farías G, Vilatroel L, Valdivia G, Mardonez JM, Díaz A. Diseño de un índice pronóstico clínico para el manejo de la neumonía del adulto adquirida en la comunidad. Rev Méd Chile 2004; 132: 37-46.

17. Cereceda J, Maturana R, Acevedo V, Aylwin M, FLORES M. Índice de gravedad en neumonía comunitaria hospitalizada. Rev Chil Enf Respir 2003; 19: 155-9.

18. Bartlett JG, Mundy LM. Current concepts: community-acquired pneumonia. N Engl J Med 1995; 33: 1618-24.

19. Gleason P, Kapoor W, Stone R, Lave J, Obrosky D, SchUIz R ET AL. Medical outcomes and antimicrobial costs with the use of the American Thoracic Society guidelines for outpatients with community-acquired pneumonia. JAMA 1997; 278: 32-9.

20. Gifason P, Meehan T, Fine J, Galusha D, Fine M. Associations between initial antimicrobial therapy and medical outcomes for hospitalized elderly patients with pneumonia. Arch Intern Med 1999; 159: 2562-72.

21. Gordon GS, Throop D, Berberian L, Niederman M, Bass J, Alemayehu D et al. Validation of the therapeutic recommendations of the American Thoracic Society (ATS) guidelines for community acquired pneumonia in hospitalized patients. Chest 1996; 110: 55S.

22. Stahl JE, Barza M, DesJardin J, Martin R, Eckman M. Effect of macrolides as part of initial empiric therapy on length of stay in patients hospitalized with community-acquired pneumonia. Arch Intern Med 1999; 159: 2576-80.

23. Lieberman D, Porath A, SchlaefFer F, Lieberman D, BowUR I. Legionella species community-acquired pneumonia. Chest 1996; 109: 1234-49.

24. Campbell S, Marrie T, Anstey R, Dickinson G, AcKroID-Stolarz S. The contribution of blood cultures to the clinical management of adult patients admitted to the hospital with community-acquired pneumonia. A prospective observational study. Chest 2003; 123: 1142-50.

25. Chalasani N, Valdecanas M, Gopal A, McGowan J, JURADO R. Clinical utility of blood cultures in adults patients with community-acquired pneumonia without defined underlying risks. Chest 1995; 108: 932-8.

26. WatereR $G$, JenNINGs $S$, Wunderink $R$. The impact of blood cultures on antibiotic therapy in pneumococcal pneumonia. Chest 1999; 116: 1278-81.

27. Díaz A, Calvo M, O’Brien A, Farías G, Mardónez J, SALDÍAS F. Utilidad clínica de los hemocultivos en pacientes hospitalizados por neumonía adquirida en la comunidad. Rev Méd Chile 2002; 130: 9931000.

28. Metersky M, Ma A, Bratzler D, Houck P. Predicting bacteremia in patients with community-acquired pneumonia. Am J Respir Crit Care Med 2004; 169: 342-7.

Agradecimientos

Los autores agradecen a Joaquim Angrill por su ayuda en el análisis estadístico y a las enfermeras de los Servicios de Medicina y Urgencia por su gran colaboración. 